\title{
AYUDAS CONCEDIDAS EN LAS CONVOCATORIAS EDUSI 2015 Y 2016 A MUNICIPIOS DE CATALUÑA: CONTEXTO Y BREVE COMENTARIO
}

\author{
Albert Lladó Martínez \\ Universitat de Girona
}

Artículo Recibido: 10/11/2017

Artículo Aceptado: 23/12/2017

\section{Resumen}

Se expone en el artículo un resumen y puesta en contexto de las ayudas otorgadas a municipios de Cataluña por las dos primeras resoluciones, de septiembre de 2016 y de mayo de 2017, de las convocatorias de Estrategias de Desarrollo Urbano Sostenible (EDUSI), cofinanciadas mediante el Programa Operativo FEDER de crecimiento sostenible 2014-2020.

La población de Cataluña se encuentra altamente concentrada en tres comarcas alrededor de la ciudad de Barcelona y esta distribución irregular se proyecta claramente en el número de solicitudes EDUSI presentadas, en la localización geográfica de estas solicitudes y del mismo modo en la de los proyectos seleccionados para su financiación.

\section{Abstract}

The article presents a summary and putting into context of the funds granted to municipalities of Catalonia by the first two resolutions, of September 2016 and May 2017, of the calls for Strategies for Sustainable Urban Development (EDUSI), cofinanced by the FEDER Operational Program for sustainable growth 2014-2020.

The population of Catalonia is highly concentrated in three districts around the city of Barcelona and this irregular distribution is clearly projected in the number of EDUSI applications submitted, in the geographic location of these applications and in the same way in the projects selected for funding.

Palabras clave: desarrollo sostenible, urbanismo, edusi, feder, cataluña, demografía.

Keywords: sustainable development, urbanism, erdf, catalonia, demography.

WPS RI-SHUR, n6, 2017, vol.2, ISSN: 2387-1768

MONOGRÁFICO SOBRE LAS ESTRATEGIAS DE DESARROLLO URBANO

SOSTENIBLE E INTEGRADO (EDUSI) 


\title{
WPSReview International on Sustainable
}

\author{
Housing and Urban Renewal (RI-SHUR)
}

\section{Las Estrategias de Desarrollo Urbano Sostenible e Integrado y el contexto geográfico y demográfico de Cataluña}

Las Estrategias de Desarrollo Urbano Sostenible e Integrado (EDUSI) responden a planificaciones diseñadas por los ayuntamientos para dar respuesta a los principales retos que ellos mismos identifican en sus correspondientes ciudades. Estas estrategias han sido presentadas por los municipios que así lo han considerado a las tres convocatorias que, hasta el momento, se han publicado para otorgar ayudas a su financiación (órdenes HAP/2427/2015, de 13 de noviembre, HAP/1610/2016, de 6 de octubre y HFP/888/2017, de 19 de septiembre).

Esta financiación tiene su origen en el Fondo Europeo de Desarrollo Regional (FEDER) de la Unión Europea, que se traduce en subvenciones a fondo perdido que serán gestionadas por las diferentes administraciones públicas, y destinadas a la realización de proyectos en los territorios de su competencia. Tal como se estipula en el Reglamento FEDER (Reglamento (UE) n.․1 1301/2013), artículo 7, durante el período 2014-2020 un mínimo de un 5\% de los recursos de este fondo deberá ser destinado a financiar medidas integradas para el desarrollo urbano sostenible, en las que las ciudades, los organismos subregionales o locales responsables de la aplicación de estrategias urbanas sostenibles serán los encargados de las tareas relacionadas, por lo menos, con la selección de las operaciones (MHAP \& MF, 2015).

Estos recursos previstos en el Reglamento FEDER encuentran su canalización en el marco del Acuerdo de Asociación de España 2014-2020, aprobado por la Comisión Europea con fecha 30 de octubre de 2014 y del Eje Urbano dentro del Programa Operativo de Crecimiento Sostenible (POCS) 2014-2020. Durante este período, los ayuntamientos y agrupaciones previstas han podido presentar sus EDUSI para optar a ser financiadas. Según lo dispuesto en el mencionado POCS, el Eje Urbano está dotado con 1.012.754.015,00 euros de ayuda FEDER y la asignación de los recursos debe realizarse a través de convocatorias abiertas, a través de las cuales se seleccionarán aquellas Estrategias DUSI que podrán ser objeto de financiación a lo largo del periodo (MHAP \& MF, 2015).

Las dos primeras convocatorias ya se han cerrado con sus correspondientes resoluciones, de septiembre de 2016 y de mayo de 2017. En Cataluña se han presentado un total de 41 solicitudes, 26 en la primera convocatoria y 15 en la segunda, y se han seleccionado 2 EDUSI en cada una de las convocatorias. Las

WPS RI-SHUR, n6, 2017, vol.2, ISSN: 2387-1768

MONOGRÁFICO SOBRE LAS ESTRATEGIAS DE DESARROLLO URBANO

SOSTENIBLE E INTEGRADO (EDUSI) 


\section{WPSReview International on Sustainable \\ Housing and Urban Renewal (RI-SHUR)}

cuatro solicitudes financiadas corresponden a municipios ubicados en un área muy concentrada alrededor de la ciudad de Barcelona.

Para poner en contexto las solicitudes y las EDUSI concedidas en estas dos primeras convocatorias, presentaré una breve descripción de la distribución de la población en Cataluña, utilizando datos referentes a 2016 del Institut d'Estadística de Catalunya, (IDESCAT, 2017).

Tabla 1 - Habitantes de Cataluña por provincias (2016)

\begin{tabular}{|l|l|l|l|l|}
\hline Cataluña & Barcelona & Girona & Lleida & Tarragona \\
\hline 7.448 .332 & 5.489 .294 & 739.607 & 428.183 & 791.248 \\
\hline & $73,7 \%$ & $9,9 \%$ & $5,7 \%$ & $10,6 \%$ \\
\hline
\end{tabular}

https://www.idescat.cat/pub/?id=aec\&n=245

Atendiendo a la primera división territorial, por provincias, se aprecia que prácticamente tres cuartas partes de la población catalana se asienta en la provincia de Barcelona, quedando el resto desigualmente repartido entre las tres provincias restantes, siendo Lleida la menos poblada con diferencia.

Por otro lado, los casi cinco millones y medio de habitantes de la provincia de Barcelona se concentran en tres comarcas alrededor de la capital: el Barcelonès, el Vallès Occidental y el Baix Llobregat.

Tabla 2 - Habitantes por comarcas de la provincia de Barcelona ${ }^{1}$ (2016)

\begin{tabular}{|l|l|l|l|}
\hline \multicolumn{2}{|l|}{ Comarcas con mayor población } & Resto comarcas \\
\hline Barcelonès & Vallès Occidental & Baix Llobregat & 7 comarcas \\
\hline
\end{tabular}

\footnotetext{
${ }^{1}$ Las divisiones provinciales y comarcales no se ajustan exactamente en referencia a los municipios que las componen. Así, por ejemplo, los municipios que integran la comarca de Osona se encuentran mayoritariamente en la provincia de Barcelona, sin embargo tres de ellos (Espinelves, Vidrà y Viladrau) pertenecen a la provincia de Girona. Lo mismo ocurre en la comarca del Berguedà con el municipio de Gòsol, perteneciente a Lleida y, en sentido contrario con Fogars de la Selva, perteneciente a la provincia de Barcelona pero dentro de una comarca de Girona.

Por este motivo, las cifras de los totales de población provinciales no coinciden exactamente con las de las sumas de la población por comarcas. Sin embargo, para los propósitos de este trabajo, la incidencia de estas pequeñas variaciones en los porcentajes de población no son significativas.
} 
WPSReview International on Sustainable

Housing and Urban Renewal (RI-SHUR)

\begin{tabular}{|l|l|l|l|}
\hline 2.205 .803 & 800.516 & \\
\hline $3.908 .839(79 \%)$ & & $1.037 .146(21 \%)$ \\
\hline
\end{tabular} Fuente de datos: Institut d'Estadística de Catalunya

https://www.idescat.cat/pub/?id=aec\&n=246

Como se comprueba en la anterior tabla 2, la población de las 7 comarcas de la provincia de Barcelona más alejadas de la capital (Alt Penedès, Anoia, Bages, Berguedà, Maresme, Moianès, Osona, Vallès Oriental) concentra únicamente alrededor del $20 \%$ del total de la provincia. Se constata, pues, la gran aglomeración de población alrededor de Barcelona, específicamente en su municipio pero también en los municipios de su alrededor, repartidos en tres comarcas.

Gráfico 1 - concentración de población alrededor de Barcelona

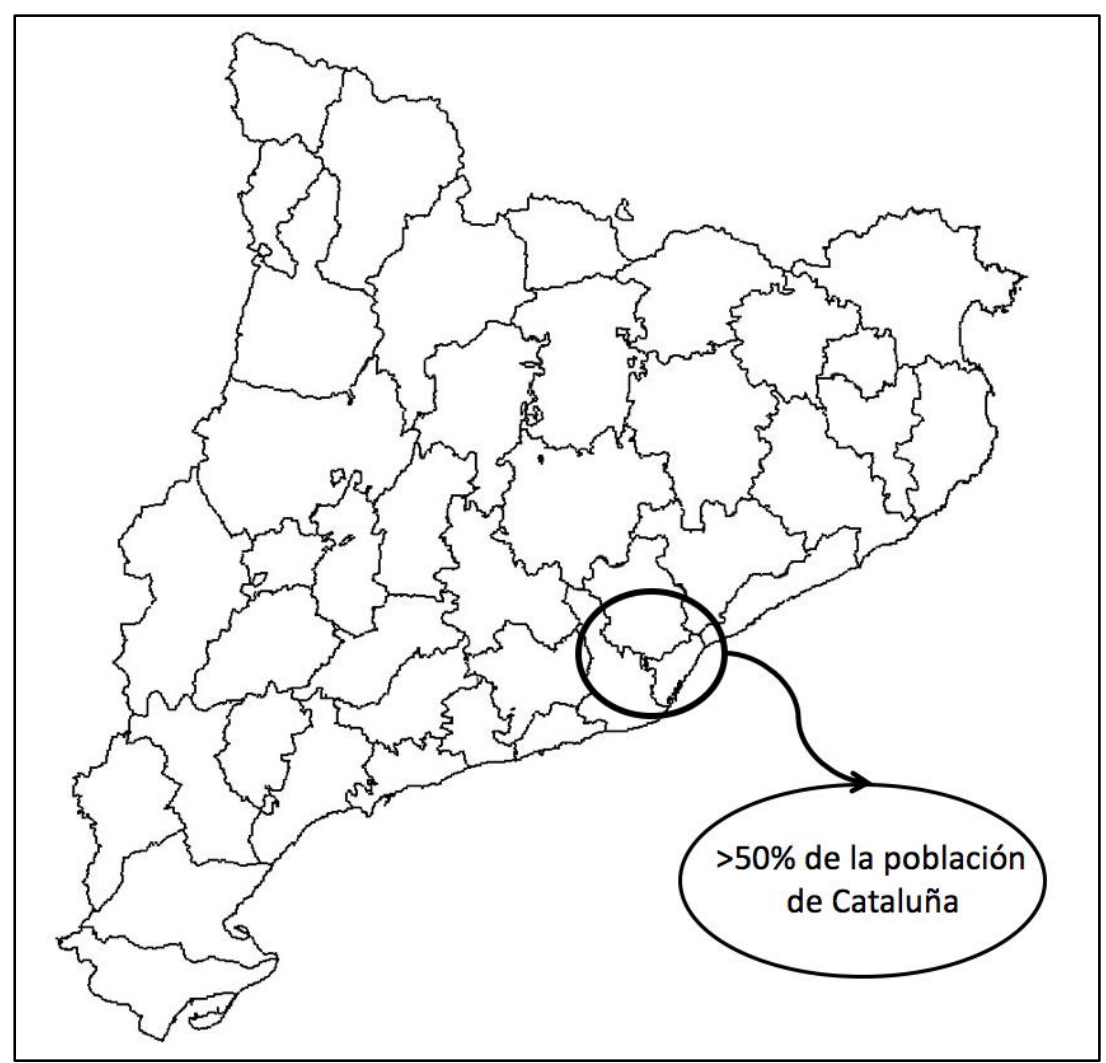

Fuente: Elaboración propia

WPS RI-SHUR, n6, 2017, vol.2, ISSN: 2387-1768

MONOGRÁFICO SOBRE LAS ESTRATEGIAS DE DESARROLLO URBANO SOSTENIBLE E INTEGRADO (EDUSI) 


\title{
WPSReview International on Sustainable
}

\author{
Housing and Urban Renewal (RI-SHUR)
}

Es necesario mencionar y diferenciar la existencia del Área Metropolitana de Barcelona (AMB), que no se corresponde exactamente con la suma de los municipios de las tres comarcas expuestas en las tablas previas. Esta entidad supramunicipal ${ }^{2}$, que ejerce competencias en cohesión, equilibrio territorial, vivienda, transporte y movilidad, ciclo del agua, residuos y medio ambiente, está compuesta por la totalidad de los municipios del Barcelonès (Barcelona, L'Hospitalet de Llobregat, Badalona, Sant Adrià de Besòs y Santa Coloma de Gramenet), pero algunos de los municipios del Baix Llobregat y del Vallès Occidental no forman parte de ella, mientras que dos localidades (Montgat y Tiana) de la comarca del Maresme, limítrofe por el norte, sí que lo hacen (AMB, 2017).

El hecho de considerar una u otra zona de referencia hace que la cantidad y la densidad de la población incluida varíen. Para el estudio y la puesta en contexto de las ayudas EDUSI, el marco de referencia es el municipio, que es la entidad que solicita estas ayudas y la que las recibe y ejecuta (si bien es cierto que la convocatoria preveía también la presentación de solicitudes por agrupaciones de municipios, las presentadas y otorgadas en Cataluña lo han sido a ayuntamientos de forma individual). Las actuaciones en el marco de cada EDUSI han de dedicarse y afectar directamente al territorio y población del municipio en cuestión, por tanto será necesario centrarse desde el punto de vista demográfico en las poblaciones de los municipios seleccionados, tanto a la hora de valorar su impacto sobre la población en términos cuantitativos como para la presente contextualización.

Sin embargo, es del todo relevante esta aproximación previa para dar un contexto claro de la forma en que está repartida la población en Cataluña, y su elevada concentración en el entorno de Barcelona. Como se evidencia en los gráficos siguientes, esta concentración se ha visto reflejada en el número de solicitudes presentadas en ambas convocatorias EDUSI y, como era esperable, las acciones seleccionadas han correspondido en todos los casos a solicitudes de municipios de esta área. En la primera resolución, las EDUSI de Santa Coloma de Gramenet y de Barcelona centraban sus actuaciones en ambas orillas del río Besòs, que es frontera natural entre los dos municipios, una zona con una densidad media de más de 20.000 habitantes por kilómetro cuadrado (IDESCAT, 2017) (AJUNTAMENT DE BARCELONA, 2017). Las EDUSI otorgadas en la segunda resolución recayeron

\footnotetext{
${ }^{2}$ Creada por la Ley del Parlament de Catalunya 31/2010, de 27 de julio, del Área Metropolitana de Barcelona.
}

WPS RI-SHUR, n6, 2017, vol.2, ISSN: 2387-1768

MONOGRÁFICO SOBRE LAS ESTRATEGIAS DE DESARROLLO URBANO

SOSTENIBLE E INTEGRADO (EDUSI) 
WPSReview International on Sustainable

Housing and Urban Renewal (RI-SHUR)

también en el entorno de la capital, en las comarcas del Vallès Occidental (Sabadell) y del Baix Llobregat (Sant Boi de Llobregat).

Gráfico 2 - situación geográfica de las ayudas EDUSI

concedidas en las dos resoluciones

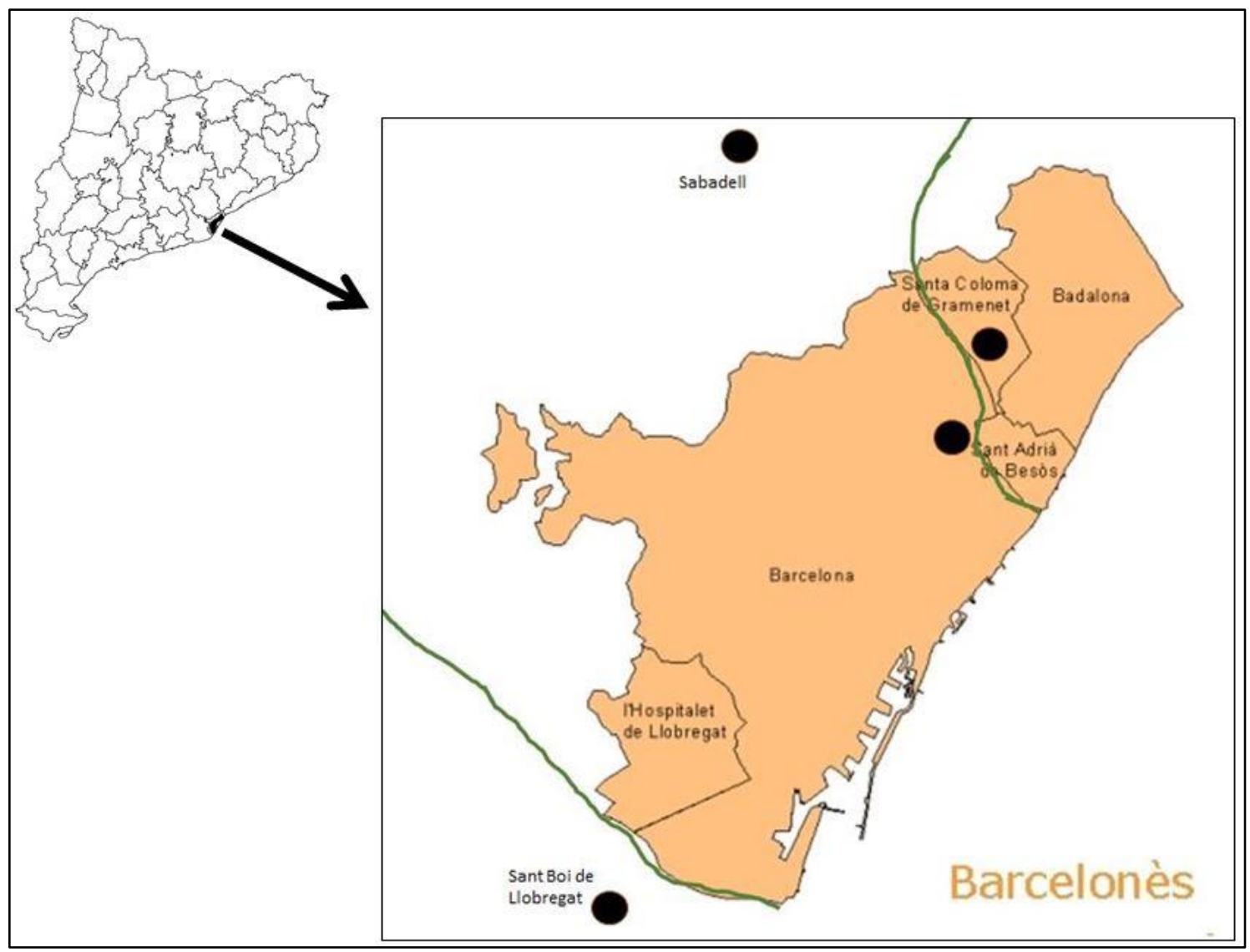

Fuente: Elaboración propia

WPS RI-SHUR, n6, 2017, vol.2, ISSN: 2387-1768

MONOGRÁFICO SOBRE LAS ESTRATEGIAS DE DESARROLLO URBANO

SOSTENIBLE E INTEGRADO (EDUSI) 


\section{WPSReview International on Sustainable \\ Housing and Urban Renewal (RI-SHUR)}

\section{Ayudas EDUSI otorgadas en Cataluña por la primera resolución, de 29 de septiembre de 2016}

La primera convocatoria se convocó, junto con las bases, en la Orden HAP/2427/2015, de 13 de noviembre, y su resolución definitiva se publicó por resolución de la Secretaría de Estado de Presupuestos y Gastos, el 12 de diciembre de 2016.

A ella se presentaron en Cataluña un total de 26 proyectos, de los cuales fueron considerados únicamente 23 , al tratarse los tres restantes de solicitudes repetidas. Por provincias, seis solicitudes fueron presentadas en Girona, dos en Tarragona y solo una en Lleida, mientras que la provincia de Barcelona concentró la gran mayoría de los proyectos, con catorce solicitudes, de las cuales dos serían concedidas.

Gráfico 3 - distribución geográfica de las solicitudes de la $1^{\underline{a}}$ convocatoria

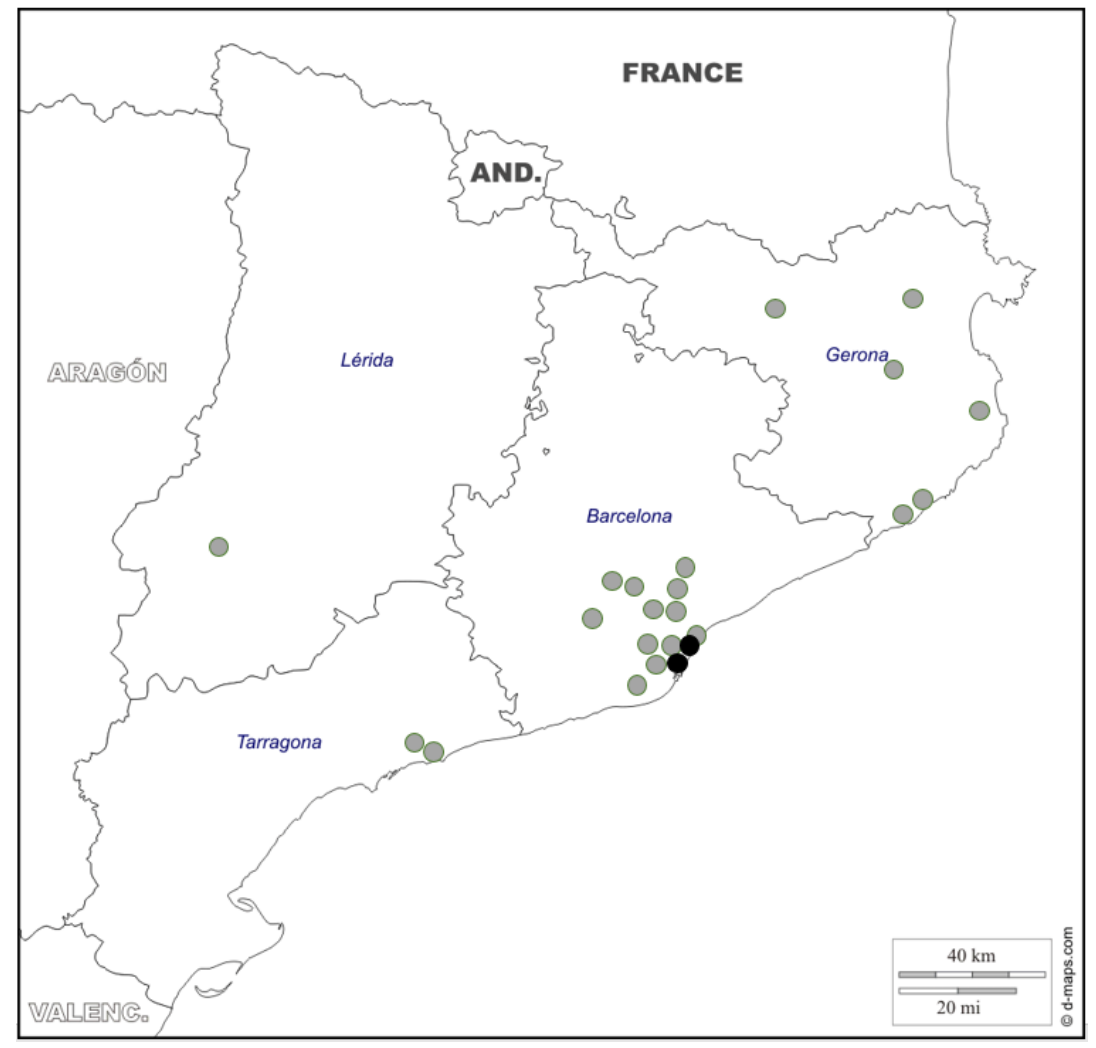

Fuente de datos: resolución MHAP de 18/05/2017. Elaboración propia.

WPS RI-SHUR, n6, 2017, vol.2, ISSN: 2387-1768

MONOGRÁFICO SOBRE LAS ESTRATEGIAS DE DESARROLLO URBANO

SOSTENIBLE E INTEGRADO (EDUSI) 


\section{WPSReview International on Sustainable \\ Housing and Urban Renewal (RI-SHUR)}

La resolución de la primera convocatoria para la selección de estrategias EDUSI cofinanciadas mediante el Programa Operativo FEDER de crecimiento sostenible, fue dictada el 29 de septiembre de 2016. Los municipios solicitantes agrupados por provincias y los resultados se reproducen en la siguiente tabla:

Tabla 3 - Municipios solicitantes en la $1^{\text {a }}$ convocatoria

\begin{tabular}{|l|l|l|}
\hline \multicolumn{1}{|c|}{ Municipios } & \multicolumn{1}{|c|}{ Provincia } & \multicolumn{1}{|c|}{ Concedidas } \\
\hline Lleida & Lleida & -- \\
\hline El Vendrell, Calafell & Tarragona & -- \\
\hline $\begin{array}{l}\text { Olot, Figueres, Blanes, Palafrugell, Girona, Lloret } \\
\text { de Mar }\end{array}$ & Girona & -- \\
\hline $\begin{array}{l}\text { Sabadell, Sta. Perpetua Mogoda, St. Vicenç dels } \\
\text { Horts, St. Boi Llobregat, Terrassa, Sta. Coloma } \\
\text { Gramenet, Barcelona, Diputación Barcelona, St. } \\
\text { Cugat Vallès, Hospitalet Llobregat, Gavà, } \\
\text { Cerdanyola Vallès, Mollet Vallès, Vilanova Camí. }\end{array}$ & & $\begin{array}{l}\text { Sta. Coloma } \\
\text { de Gramenet, } \\
\text { Barcelona. }\end{array}$ \\
\hline
\end{tabular}

Fuente de datos: resolución MHAP de 29/09/2016. Elaboración propia

Las solicitudes concedidas fueron las presentadas por los Ayuntamientos de Santa Coloma de Gramenet y de Barcelona. El resto fueron descartadas por no haber obtenido suficiente puntuación, excepto en el caso de la solicitud del Ayuntamiento de Vilanova del Camí que no era elegible por tener una población menor de veinte mil habitantes.

Las dos ayudas otorgadas fueron solicitadas en solitario por los respectivos ayuntamientos y en ambos casos obtuvieron la ayuda máxima prevista para su tipología, 15 millones de euros provenientes del Programa Operativo de Crecimiento Sostenible FEDER 2014-2020.

2.1 EDUSI seleccionada del Ayuntamiento de Santa Coloma de Gramenet.

Santa Coloma de Gramenet es un municipio perteneciente a la comarca del Barcelonès que, por el suroeste, comparte el río Besòs como límite con la ciudad de

WPS RI-SHUR, n6, 2017, vol.2, ISSN: 2387-1768

MONOGRÁFICO SOBRE LAS ESTRATEGIAS DE DESARROLLO URBANO SOSTENIBLE E INTEGRADO (EDUSI) 


\section{WPSReview International on Sustainable \\ Housing and Urban Renewal (RI-SHUR)}

Barcelona. La densidad de población de Sta. Coloma es de 16.736 hab $/ \mathrm{km}^{2}$, según datos del año 2016 del IDESCAT, a partir de un total de 117.153 habitantes repartidos en una superficie de $7 \mathrm{~km}^{2}$.

La EDUSI del Ayuntamiento de Santa Coloma de Gramenet se inscribe en la tipología 1 del Anexo I de la convocatoria de noviembre de 2015, esto es, presentada por un único municipio con una población mayor de 20.000 habitantes. Esta tipología permite estrategias referidas a la totalidad del municipio o, como es el caso, limitadas a "áreas urbanas inframunicipales de diferente tipología morfológica y funcional: centros históricos, barrios desfavorecidos o áreas suburbanas con problemáticas específicas y retos singulares", según se estipula en la propia convocatoria. En este caso la EDUSI no incluye una limitación geográfica, aunque sí que se prevén actuaciones en barrios y zonas concretas de la ciudad.

En la estrategia se fijan unos valores iniciales y finales esperados para una serie de indicadores de productividad, contemplados en el plan de implementación, y que van a ser monitorizados a la finalización del plan para comprobar la consecución de los valores esperados. Los indicadores son (MHAP, 2015):

-Reducción del consumo final de energía

-Número de usuarios cubiertos por la administración electrónica

-Número de usuarios cubiertos por la Smart City

-Beneficiarios de la regeneración física, económica y social

-Superficie de edificios patrimonio cultural rehabilitados

-Número de visitas al patrimonio cultural

-Superficie total de suelo rehabilitado

-Reducción anual de gases de efecto invernadero

-Reducción del consumo anual de energía primaria en edificios públicos

La incidencia sobre estos indicadores, se prevé conseguir mediante el trabajo sobre una serie de retos que se afrontarán durante el proyecto:

-Implantación de Smart City

-Mejora de la comunicación con Barcelona y con el Área Metropolitana

-Mejora de la urbanización del centro de la ciudad y de espacios públicos

-Ampliación de zonas verdes

-Reducción del tráfico rodado

-Actuación sobre el parque fluvial del Besòs

-Apuesta por las energías renovables

WPS RI-SHUR, n6, 2017, vol.2, ISSN: 2387-1768

MONOGRÁFICO SOBRE LAS ESTRATEGIAS DE DESARROLLO URBANO

SOSTENIBLE E INTEGRADO (EDUSI) 


\title{
WPSReview International on Sustainable
}

\section{Housing and Urban Renewal (RI-SHUR)}

\author{
-Modernización y remodelación de los mercados municipales \\ -Promoción turística de la ciudad \\ -Creación de viveros de empresas \\ -Extensión de la universidad al conjunto de la ciudad
}

Los ámbitos cubiertos por las líneas concretas de actuación se ordenan en cuatro objetivos temáticos (OT), que son los contemplados en el Anexo VII de la convocatoria, en coherencia con el Programa Operativo de Crecimiento Sostenible. Las líneas de actuación previstas en la EDUSI son las siguientes:

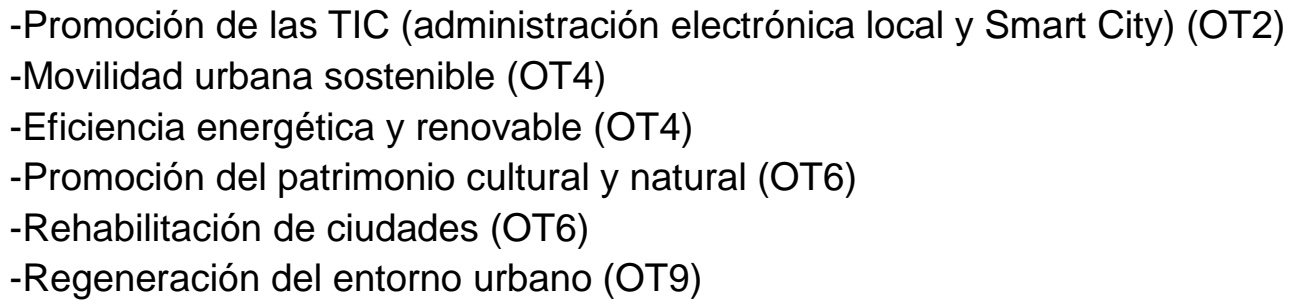

Un ejemplo de actuación ya iniciada, que integra diversos de los ámbitos de incidencia previstos, es la que parcialmente ya se ha llevado a cabo para el nuevo diseño del Paseo de la Salzereda, que cubre la mayor parte de la fachada de la ciudad a lo largo del río Besòs. Se aúnan en ella actuaciones en rehabilitación y regeneración urbana, así como en movilidad sostenible y promoción del patrimonio natural. Además, se trata de una actuación en la cual se ha promovido notablemente la participación ciudadana, con la presentación en diversas reuniones vecinales promovidas por el Ayuntamiento de dos propuestas diferentes para la remodelación del paseo. El debate promovido entre las dos opciones culminó con una consulta participativa, en la cual los vecinos afectados decidieron la opción preferida, que será desarrollada efectivamente durante el período de implementación de la EDUSI.

\subsection{EDUSI seleccionada del Ayuntamiento de Barcelona}

De la misma forma que la anterior, la EDUSI del Ayuntamiento de Barcelona (AJUNTAMENT DE BARCELONA, 2014) forma parte de las previstas en la tipología 1 del Anexo I de la convocatoria de noviembre de 2015, al ser presentada por un único municipio con una población mayor de 20.000 habitantes. En este caso, la estrategia no va dirigida a la totalidad del municipio sino que se limita geográficamente como se prevé en la convocatoria a "áreas urbanas inframunicipales de diferente tipología

WPS RI-SHUR, n6, 2017, vol.2, ISSN: 2387-1768

MONOGRÁFICO SOBRE LAS ESTRATEGIAS DE DESARROLLO URBANO SOSTENIBLE E INTEGRADO (EDUSI) 


\section{WPSReview International on Sustainable \\ Housing and Urban Renewal (RI-SHUR)}

morfológica y funcional: centros históricos, barrios desfavorecidos o áreas suburbanas con problemáticas específicas y retos singulares".

El ámbito de actuación concreto comprende diez barrios del municipio de Barcelona que pertenecen a tres distritos, todos ellos colindantes con el cauce del río Besòs:

- Distrito de Noubarris: comprende los barrios de Vallbona, Ciutat Meridiana, Torre Baró, Roquetes, Trinitat Nova.

- Distrito de Sant Andreu: comprende los barrios de Trinitat Vella, Baró de Viver, Bon Pastor.

- Distrito de Sant Martí: comprende los barrios de Verneda-La Pau, Maresme-Besòs.

Tabla 4 - Población de los distritos objeto de la EDUSI de Barcelona (2015)

\begin{tabular}{|l|l|l|l|}
\hline Distrito & Habitantes & Superficie $\left(\mathrm{km}^{2}\right)$ & $\begin{array}{l}\text { Densidad } \\
\left(\mathrm{hab}\left(\mathrm{km}^{2}\right)\right.\end{array}$ \\
\hline Nou Barris & 164.648 & 8,04 & 20.478 \\
\hline Sant Andreu & 146.494 & 6,57 & 22.297 \\
\hline Sant Martí & 233.659 & 10,52 & 22.210 \\
\hline \multicolumn{2}{|l|}{ Densidad media de la ciudad de Barcelona } & 15.706 \\
\hline
\end{tabular}

Fuente de datos: Departamento de Estadística del Ayuntamiento de Barcelona: http://www.bcn.cat/estadistica/castella/dades/anuari/cap01/C0101050.htm)

La elección de este ámbito concreto se justifica en la propia memoria presentada por el Ayuntamiento (AJUNTAMENT DE BARCELONA, 2014), que se basa en la previa elaboración de un Plan Estratégico Piloto de intervención social integrada en la denominada "Franja Besòs de Barcelona", en la cual, según el propio documento, se detectan grandes desigualdades respecto a la media de la ciudad en un buen número de los indicadores en los ámbitos de población, economía y sociedad, que incluyen:

-Porcentaje de inmigración

-Esperanza de vida al nacer

-Índice de envejecimiento

-Índice de sobreenvejecimiento

-Hogares monoparentales

-Hogares con +7 habitantes

-Renta familiar disponible

-Paro (\%) total, hombres, mujeres, larga duración

-Población sin estudios

WPS RI-SHUR, n6, 2017, vol.2, ISSN: 2387-1768

MONOGRÁFICO SOBRE LAS ESTRATEGIAS DE DESARROLLO URBANO

SOSTENIBLE E INTEGRADO (EDUSI) 


\section{WPSReview International on Sustainable}

Housing and Urban Renewal (RI-SHUR)

-Población con estudios superiores

-Población con discapacidad

-Precio vivienda segunda mano

-Población de +65 que vive sola

-Pensionistas de +65 (no contributiva)

-Personas de +65 atendidas por teleasistencia

Para incidir de forma positiva en estos indicadores, la estrategia identifica una serie de retos a los cuales se prevé hacer frente mediante el proyecto:

-Equilibrar la brecha digital a valores referencia de la ciudad

-Disminuir el modo coche del reparto modal

-Mejorar y fomentar el Besòs como espacio de calidad ambiental y patrimonial

-Aumentar la resiliencia frente al cambio climático

-Disminuir el fracaso y abandono escolar prematuros equilibrándolos con el resto de la ciudad

-Disminuir el número de personas en paro equilibrándolo con el resto de la ciudad

- Incrementar el desarrollo económico basado en comercio de proximidad, economía circular, social, y/o tecnológica y autocontención

-Aumentar las rentas familiares equilibrándolas con el resto de la ciudad

-Disminuir el número de personas en riesgo de exclusión social equilibrándola con el resto de la ciudad

-Aumentar el número de personas que practican deportes y una forma de vida saludable

-Mejorar la calidad de vida de las personas mayores

Finalmente, se especifican una serie de líneas de actuación sobre las cuales se incidirá con las acciones previstas en la EDUSI. Los ámbitos cubiertos por estas líneas se ordenan en los cuatro objetivos temáticos que se contemplan en el Anexo VII de la convocatoria, en coherencia con el Programa Operativo de Crecimiento Sostenible:

-Tecnología para la integración (OT2)

-Fomento de la tecnología (OT2)

-Fomento de la movilidad a pie y en bici (OT4)

-Plan de movilidad polígono industrial y comercial (OT4)

- Implantar medidas de urbanización que reduzcan el tráfico de vehículos privados y mejoren la dedicación del espacio público a la movilidad sostenible y a la vida cotidiana (OT4)

WPS RI-SHUR, n6, 2017, vol.2, ISSN: 2387-1768

MONOGRÁFICO SOBRE LAS ESTRATEGIAS DE DESARROLLO URBANO

SOSTENIBLE E INTEGRADO (EDUSI) 


\section{WPSReview International on Sustainable \\ Housing and Urban Renewal (RI-SHUR)}

-La memoria del agua. Acceso y uso cívico del río (OT6)

- Incentivar la formación y la empleabilidad en el área urbana (OT9)

-Implementar planes de desarrollo local para fomentar la actividad económica y ocupación (OT9)

-Refuerzo de los servicios sociales a los colectivos en riesgo de exclusión (OT9)

- Mejorar el acceso y la accesibilidad a la vivienda (OT9)

La temporalización de las actuaciones se encuentra prevista a lo largo de los ejercicios 2017, 2018 y 2019, y el propio documento referenciado incluye fichas descriptivas detalladas para cada una de las actuaciones previstas. En este sentido, es destacable que el Ayuntamiento facilite en acceso abierto la memoria de la EDUSI para la cual ha obtenido financiación, hecho que desafortunadamente no es práctica habitual en la mayoría de casos.

\section{Ayudas EDUSI otorgadas en Cataluña por la segunda resolución, de 18 de mayo de 2017}

La segunda convocatoria de ayudas a las EDUSI fue publicada mediante la Orden HAP/1610/2016, de 6 de octubre y se resolvió definitivamente por resolución de la Secretaría de Estado de Presupuestos y Gastos el 18 de mayo de 2017.

La concurrencia de proyectos en Cataluña fue en esta ocasión bastante menor que en la primera convocatoria. Esta vez se presentaron un total de 15 solicitudes, de las cuales tres fueron presentadas en la provincia de Girona, una en Tarragona, una en Lleida y diez en la provincia de Barcelona, localizadas una vez más en los municipios de la Área Metropolitana de Barcelona.

WPS RI-SHUR, n6, 2017, vol.2, ISSN: 2387-1768

MONOGRÁFICO SOBRE LAS ESTRATEGIAS DE DESARROLLO URBANO

SOSTENIBLE E INTEGRADO (EDUSI) 
WPSReview International on Sustainable

Housing and Urban Renewal (RI-SHUR)

Gráfico 4 - distribución geográfica de las solicitudes de la $2^{\mathrm{a}}$ convocatoria

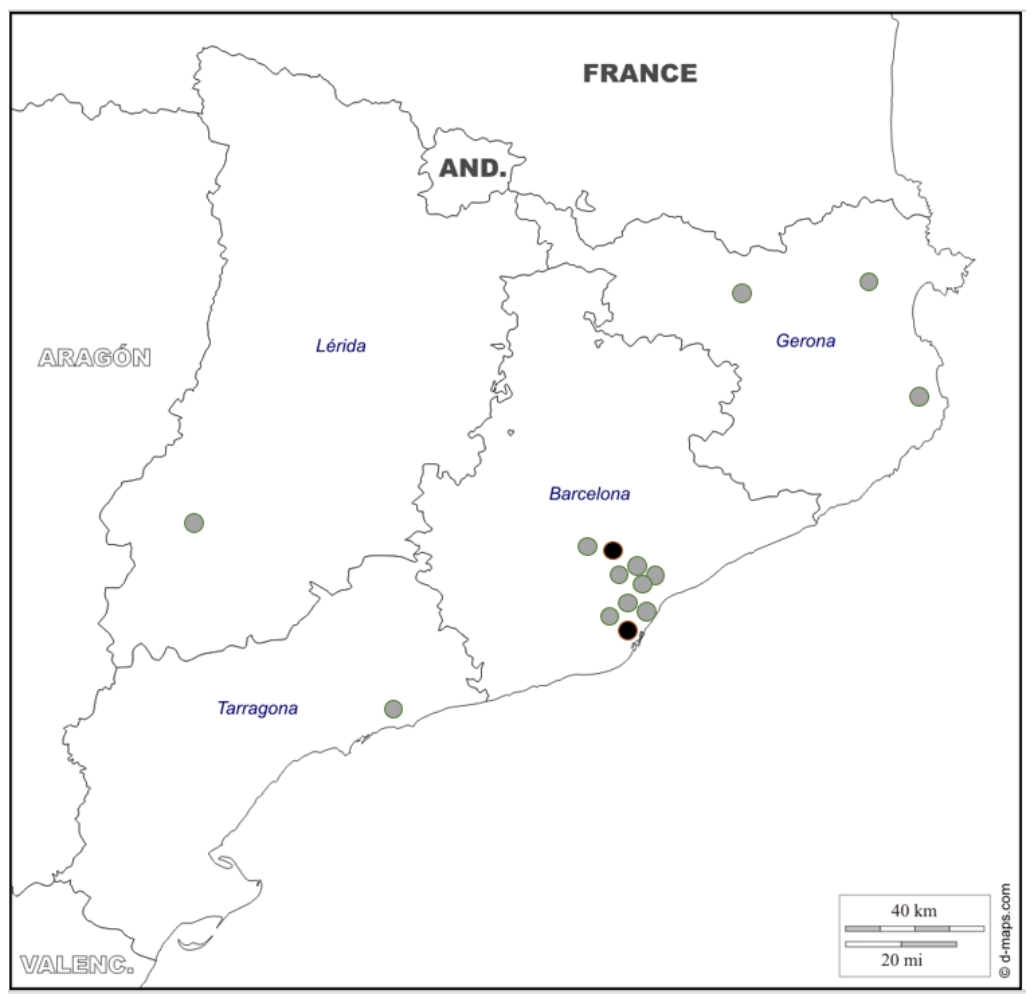

Fuente de datos: resolución MHAP de 18/05/2017. Elaboración propia

La resolución de esta segunda convocatoria se dictó el 18 de mayo de 2017, con los resultados siguientes en Cataluña (en negrita los municipios que no presentaron candidatura en la convocatoria anterior):

Tabla 5 - Municipios solicitantes en la $2^{\underline{a}}$ convocatoria

\begin{tabular}{|l|l|l|}
\hline Municipios & Provincia & Concedidas \\
\hline Lleida & Lleida & -- \\
\hline El Vendrell & Tarragona & -- \\
\hline Olot, Figueres, Palafrugell & Girona & -- \\
\hline $\begin{array}{l}\text { Sabadell, Esplugues Llobregat, Sta. Perpetua } \\
\text { Mogoda, St. Vicenç dels Horts, St. Boi Llobregat, } \\
\text { Terrassa, St. Cugat Vallès, Hospitalet Llobregat, } \\
\text { Cerdanyola Vallès, Montcada i Reixach }\end{array}$ & & $\begin{array}{l}\text { Sabadell, St. } \\
\text { Boi Llobregat }\end{array}$ \\
\hline
\end{tabular}

Fuente de datos: resolución MHAP de 18/05/2017. Elaboración propia

WPS RI-SHUR, n6, 2017, vol.2, ISSN: 2387-1768

MONOGRÁFICO SOBRE LAS ESTRATEGIAS DE DESARROLLO URBANO

SOSTENIBLE E INTEGRADO (EDUSI) 


\section{WPSReview International on Sustainable \\ Housing and Urban Renewal (RI-SHUR)}

\subsection{EDUSI seleccionada del Ayuntamiento de Sabadell.}

La ciudad de Sabadell es capital de la comarca del Vallès Occidental, juntamente con Terrassa. Según datos del IDESCAT, su población se cifra (2016) en 208.246 personas, distribuidas en una superficie de $37,79 \mathrm{~km}^{2}$, lo que supone una densidad de $5.510,6 \mathrm{hab} / \mathrm{km}^{2}$.

La EDUSI fue presentada por un único municipio con una población mayor de 20.000 habitantes, por tanto forma parte de las previstas en la tipología 1 del Anexo I de las bases publicadas con la convocatoria de noviembre de 2015. La estrategia y su aplicación están dirigidas a la totalidad del municipio y de su población, con actuaciones específicas en áreas y colectivos concretos.

Los indicadores utilizados para identificar los aspectos sobre los cuales la EDUSI se iba a diseñar, toman como base aspectos de la vida urbana en la ciudad (AJUNTAMENT DE SABADELL, 2015). A través de ellos se detectan ámbitos en que Sabadell presenta un diferencial comparativo respecto de otras ciudades del entorno nacional o europeo. La EDUSI se refiere a los aspectos estudiados como problemas urbanos, y aquellos identificados como más problemáticos y necesitados de actuación son los siguientes:

Problema urbano 1:

-Riesgo de fractura digital en ciertos grupos sociales, por edad o por nivel socioeconómico.

-Dificultad para conciliar las necesidades de mantenimiento ordinario con la incorporación de las TIC innovadoras a los servicios urbanos.

-Visión negativa de la ciudadanía respecto del grado de transparencia y ética de los poderes públicos.

Problema urbano 2:

-Muy bajo nivel de producción local de energía renovable.

- La ciudad supera alguno de los niveles máximos permitidos de contaminación ambiental.

-Alta proporción de movilidad privada contaminante.

-Alta proporción de edificios construidos sin considerar criterios de eficiencia energética.

Problema urbano 3:

-Dificultad de diseñar modelos de rentabilidad económica privada para el aprovechamiento agrario del Rodal periurbano.

WPS RI-SHUR, n6, 2017, vol.2, ISSN: 2387-1768

MONOGRÁFICO SOBRE LAS ESTRATEGIAS DE DESARROLLO URBANO

SOSTENIBLE E INTEGRADO (EDUSI) 


\section{WPSReview International on Sustainable \\ Housing and Urban Renewal (RI-SHUR)}

-Inframantenimiento del espacio público por el decrecimiento del presupuesto municipal.

- Infraestructuras físicas suponen barreras a la movilidad no motorizada.

-Existencia de edificios o solares vacíos o abandonados con un uso primitivo como actividad económica, algunos de ellos con valor patrimonial.

-Contaminación acústica vinculada a la movilidad o a los usos de ocio.

Problema urbano 4:

-Alto grado de fracaso escolar y población con estudios inferiores a los secundarios.

-Elevado nivel de paro y bajo nivel de población activa.

-Riesgo de envejecimiento de la población.

-Diferencias socio-económicas importantes entre barrios.

-Presupuesto municipal insuficiente para afrontar inversiones o gastos más allá del estricto mantenimiento ordinario.

-Falta de una estrategia clara de desarrollo económico.

-Dificultad para atraer o retener población joven y empresas vinculadas a la I+D y lugares de trabajo de calificación mediana y alta.

Los retos identificados a partir de los indicadores expuestos son enmarcados en la EDUSI alrededor de las temáticas económica, ambiental, climática, demográfica y social:

Retos económicos:

-La reindustrialización y el cambio de modelo productivo como mecanismo para la reactivación económica.

-Asegurar y complementar la capacidad de inversión y la estabilidad financiera del Ayuntamiento.

Retos ambientales:

-Sostenibilidad, eficiencia energética y calidad del espacio urbano.

-Calidad del aire y espacio urbano saludable: una ciudad para peatones y bicicletas.

Retos climáticos:

- Una ciudad hacia las emisiones cero.

Retos demográficos:

-El hábitat y la vivienda como estructuradores de la ciudad.

-Envejecimiento de la población.

Retos sociales:

-La energía como servicio básico.

-TIC y ciudadanía: la transparència i la participació como forma de gobierno.

WPS RI-SHUR, n6, 2017, vol.2, ISSN: 2387-1768

MONOGRÁFICO SOBRE LAS ESTRATEGIAS DE DESARROLLO URBANO

SOSTENIBLE E INTEGRADO (EDUSI) 


\section{WPSReview International on Sustainable \\ Housing and Urban Renewal (RI-SHUR)}

Las líneas de actuación previstas se enmarcan en los cuatro objetivos temáticos contemplados en el Anexo VII de la convocatoria, en consonancia con lo previsto por el Programa Operativo de Crecimiento Sostenible:

OT2:

-Promover a través de las TIC el impulso del comercio de proximidad, el turismo y el encuentro empresarial.

- Implantar la tarjeta ciudadana.

- Implantar una aplicación móvil para comunicar las incidencias en la vía pública.

- Impulsar las herramientas TIC para la transparencia y la nueva gobernanza.

- Ofrecer una administración electrónica accesible.

- Mejorar la tecnología en la prestación de servicios municipales.

-Desarrollar la plataforma "Open Data" para compartir datos entre diversos actores urbanos.

-Desarrollar la plataforma "Open Data \& Big Data" a través del programa de GIS.

-Optimizar el sistema de gestión energética de los edificios municipales.

-Control inteligente de la gestión del ciclo del agua.

-Control inteligente de la gestión de la flota del transporte público urbano.

OT4:

-Ampliación de los recorridos a pie en ámbito urbano y periurbano.

- Completar y finalizar la red urbana e interurbana para bicicletas.

- Incorporar a la flota de autobuses vehículos $100 \%$ eléctricos.

- Gestión eficiente de la distribución urbana de mercancías.

-Rehabilitar energéticamente el Parque Público de Viviendas HPO en alquiler.

- Rehabilitar energéticamente equipamientos municipales y públicos.

- Implantar energías renovables en edificios públicos.

-Consolidar un espacio como Fábrica-Laboratorio en la oficina de la energía.

OT6:

-Repatrimonializar la ciudad a través de la adquisición de bienes singulares de gran valor patrimonial.

- Impulsar el modelo productivo de agricultura de proximidad.

-Creación de infraestructuras y espacios verdes conectores de tejidos urbanos para la cohesión social.

-Implantar Zonas Urbanas Pacificadas y Tranquilas.

OT9:

WPS RI-SHUR, n6, 2017, vol.2, ISSN: 2387-1768

MONOGRÁFICO SOBRE LAS ESTRATEGIAS DE DESARROLLO URBANO

SOSTENIBLE E INTEGRADO (EDUSI) 


\section{WPSReview International on Sustainable \\ Housing and Urban Renewal (RI-SHUR)}

-Reconversión del patrimonio en espacios estratégicos para la innovación empresarial y coworking para proyectos impulsados por menores de 35 años o por empresas de amplio espectro de inclusión social.

-Creación de espacios para creadores: viveros empresariales con nuevos modelos de emprendimiento y economía social.

-Creación de la Oficina de la Energía Municipal en Campoamor y Espronceda.

-Impulso de colaboraciones entre la Estrategia DUSI y la red universitaria, FP y escuela en barrios emergentes.

-Pedagogía de la Sostenibilidad: Caminos escolares en sectores populares de la ciudad - Can Deu, Sant Julián y la Planada.

-Impulsar el deporte como herramienta de cohesión social.

-Rehabilitar el patrimonio municipal de viviendas como Fondo de Emergencia Social.

-Implantar la Agencia Municipal de Alquiler para el alquiler de viviendas desocupadas y/o en venta.

\subsection{EDUSI seleccionada del Ayuntamiento de Sant Boi de Llobregat.}

Sant Boi de Llobregat es un municipio de la comarca del Baix Llobregat, situada justo al sur de la del Barcelonès; como la mayoría de los municipios de esta comarca, Sant Boi forma parte del Área Metropolitana de Barcelona.

A partir de sus datos básicos demográficos (IDESCAT, 2017), el municipio tiene una población según datos de 2016 de 82.402 ciudadanos y una superficie de 21,47 kilómetros cuadrados. La densidad de población es, por tanto, de 3.838 hab/km².

Se trata de una EDUSI de tipología 1, presentada por un único municipio y dirigida a actuar en la totalidad de su territorio. Sin embargo, su ámbito de actuación se define en una doble dirección (AJUNTAMENT DE SANT BOI DE LLOBREGAT, 2016), con una parte de la estrategia focalizada en dos barrios periféricos: Camps Blancs y Ciutat Cooperativa-Molí Nou. Este ámbito de actuación se complementa con las actuaciones dirigidas a la ciudad en general.

Los indicadores utilizados para identificar los principales problemas del municipio se obtuvieron a partir de un análisis del conjunto del área urbana, realizado desde una perspectiva integradora, que se centró en los siguientes ámbitos:

WPS RI-SHUR, n6, 2017, vol.2, ISSN: 2387-1768

MONOGRÁFICO SOBRE LAS ESTRATEGIAS DE DESARROLLO URBANO

SOSTENIBLE E INTEGRADO (EDUSI) 


\section{WPSReview International on Sustainable \\ Housing and Urban Renewal (RI-SHUR)}

-Contexto territorial, Contexto físico, Ambiental y de condiciones climáticas, Energético, Económico, Demográfico, Social, de Conflictividad, de la Planificación existente, del Marco competencial y de Riesgos.

A partir de estos análisis, se identificaron una serie ámbitos en los cuales se insertan los retos que será necesario afrontar. Los ámbitos se distribuyen en retos sociales, económicos, ambientales, climáticos y de gobernanza y dentro de ellos, se focaliza en seis grandes problemas que se pretenden abordar:

-Conectividad funcional y desconexión del entorno natural.

- Tejido productivo de escaso valor añadido.

-Envejecimiento demográfico.

-Desempleo y necesidades de formación.

-Vulnerabilidad e ineficiencia energética.

-Implantación insuficiente de las TIC en la gestión y servicios a la ciudadanía.

En el apartado de las líneas de actuación propuestas, las acciones financiables previstas en la EDUSI son las siguientes, asociadas al Objetivo Temático correspondiente:

-Conectar con fibra óptica el Ayuntamiento con todos los equipamientos municipales de la ciudad e instalación de wi-fi en todos ellos (OT2)

-Desarrollo del e-gobierno (OT2)

-Desarrollo de la e-administración (OT2)

-Mejora de la movilidad mediante la instalación de sensores, ampliación de la red de fibra óptica y diseño de aplicaciones (OT4)

-Rehabilitación energética de edificios públicos y privados (OT4)

-Ampliación del camino natural del "Anell Verd" mediante restauración ambiental de la zona "Torre de la Vila" (OT6)

-Rehabilitación del Palacio de Marianao para acoger el centro de formación e impulso de proyectos relacionados con la salud mental (OT6)

-Estrategia de comunicación y promoción de los valores y potenciales de la ciudad: entorno natural, patrimonio cultural y trama urbana de convivencia ciudadana (OT6)

-Espacio polivalente para un centro social de personas mayores y una incubadora de iniciativas de emprendeduría y economía social (OT9)

-Revitalización del espacio público de Cooperativa-Molí Nou: reurbanización de los espacios entre bloques y la creación de ejes verdes (OT9)

-Atención a las personas mayores y en situación de dependencia (OT9)

WPS RI-SHUR, n6, 2017, vol.2, ISSN: 2387-1768

MONOGRÁFICO SOBRE LAS ESTRATEGIAS DE DESARROLLO URBANO

SOSTENIBLE E INTEGRADO (EDUSI) 


\title{
WPSReview International on Sustainable
}

\author{
Housing and Urban Renewal (RI-SHUR)
}

-Inserción laboral de jóvenes y otros colectivos con dificultades de acceso al mercado laboral (OT9)

-Estrategia de excelencia educativa, éxito escolar e innovación educativa (OT9)

-Fomento para el acceso a la vivienda a jóvenes y personas en riesgo de exclusión social (OT9)

\section{Resultados y Conclusiones}

- Existe una alta concentración de la población de Cataluña en el área próxima a la ciudad de Barcelona (comarcas del Barcelonès, Vallès Occidental y Baix Llobregat). La mayoría de grandes ciudades se encuentran en esta área (14 del total de 23 ciudades de más de 50.000 habitantes existentes en Cataluña), y la gran mayoría de las solicitudes de ayudas a la financiación de las EDUSI (un 63\%) han sido presentadas por municipios de estas comarcas.

- Los resultados de las adjudicaciones de ayudas en las dos primeras resoluciones han sido aún más drásticos: el total de las cuatro ayudas a EDUSI que se han concedido han sido otorgadas a municipios de esta zona geográfica: Barcelona y Santa Coloma de Gramenet en la primera resolución, de la comarca del Barcelonès, y en la segunda resolución Sabadell (Vallès Occidental) y Sant Boi de Llobregat (Baix Llobregat).

- Los municipios que se han presentado a las convocatorias lo han hecho en solitario, dentro de la tipología 1 del Anexo I de las bases incluidas en la convocatoria de noviembre de 2015. Han sido presentadas por tanto por un único municipio con una población mayor de 20.000 habitantes. No se han solicitado ayudas para estrategias DUSI en las restantes modalidades previstas en el mismo Anexo (modalidad 2, agrupaciones de municipios con una población de cada uno de ellos superior a 20.000 habitantes; modalidad 3, áreas urbanas formadas por un municipio o conurbación de más de 20.000 habitantes y municipios periféricos de menos de 20.000 habitantes y modalidad 4, conurbaciones de población superior a 20.000 habitantes, constituidas por agrupaciones de municipios de menos de 20.000 habitantes).

\section{Referencias bibliográficas}

\section{AJUNTAMENT DE BARCELONA. (2014). Estrategia de Desarrollo Urbano Sostenible e Integrado 2014-2020. Barcelona. Retrieved from http://ajuntament.barcelona.cat/ecologiaurbana/sites/default/files/EDUSI_Barcelon}

WPS RI-SHUR, n6, 2017, vol.2, ISSN: 2387-1768

MONOGRÁFICO SOBRE LAS ESTRATEGIAS DE DESARROLLO URBANO

SOSTENIBLE E INTEGRADO (EDUSI) 
WPSReview International on Sustainable

Housing and Urban Renewal (RI-SHUR)

a_2014_2020.pdf

AJUNTAMENTT DE BARCELONA. (2017). Web Ajuntament de Barcelona. Retrieved October 25, 2017, from http://www.bcn.cat/estadistica/catala/dades/anuari/cap02/C020104.htm

AJUNTAMENT DE SABADELL. (2015). Projecte estratègia DUSI Sabadell. Sabadell. Retrieved from http://www.consensus.cat/sabadell/components/com_docs/files/Document final EDUSI Sabadell-1.pdf

AJUNTAMENT DE SANT BOI DE LLOBREGAT. (2016). Estrategia DUSI Sant Boi de Llobregat 2016. Sant Boi de Llobregat. Retrieved from http://www.santboi.cat/fotos/dusi/DUSI Sant Boi 2016.pdf

AMB. (2017). Web Àrea Metropolitana de Barcelona. Retrieved October 24, 2017, from http://www.amb.cat/s/home.html

IDESCAT. (2017). Web Institut d'Estadística de Catalunya. Retrieved October 23, 2017, from http://www.idescat.cat

MHAP. (2015). Ficha resumen EDUSI Santa Coloma Gramenet. Madrid. Retrieved from

http://www.rediniciativasurbanas.es/iniciativas.web/static/edusi/EDUSI_201638.pd f

Convocatorias y resoluciones:

-Orden HAP/2427/2015, de 13 de noviembre, por la que se aprueban las bases y primera convocatoria de ayudas EDUSI: https://www.boe.es/boe/dias/2015/11/17/pdfs/BOE-A-2015-12423.pdf

-Orden HAP/1610/2016, de 6 de octubre, por la que se aprueba la segunda convocatoria de ayudas EDUSI: https://www.boe.es/boe/dias/2016/10/07/pdfs/BOE-A2016-9222.pdf

-Orden HFP/888/2017, de 19 de septiembre, por la que se aprueba la tercera convocatoria de ayudas EDUSI: https:/www.boe.es/boe/dias/2017/09/21/pdfs/BOE-A2017-10772.pdf

-Resolución de la primera convocatoria: https://www.boe.es/boe/dias/2016/10/03/pdfs/BOE-A-2016-9052.pdf

-Resolución de la segunda convocatoria:

https://www.boe.es/boe/dias/2017/07/25/pdfs/BOE-A-2017-8814.pdf

$\begin{array}{ll}\text { Abreviaturas } & \\ \text { AMB } & \text { Área Metropolitana de Barcelona } \\ \text { EDUSI } & \text { Estrategia de Desarrollo Urbano Sostenible e Integrado } \\ \text { FEDER } & \text { Fondo Europeo de Desarrollo Regional } \\ \text { IDESCAT } & \text { Institut d'Estadística de Catalunya } \\ \text { MHAP } & \text { Ministerio de Hacienda y Administraciones Públicas } \\ \text { OT } & \text { Objetivo Temático }\end{array}$

WPS RI-SHUR, n6, 2017, vol.2, ISSN: 2387-1768

MONOGRÁFICO SOBRE LAS ESTRATEGIAS DE DESARROLLO URBANO

SOSTENIBLE E INTEGRADO (EDUSI) 